\section{Recovery of the Resistivity of Metals after Cold-Working}

Is communications in Nature ${ }^{1,2}$, measurements have been described on the recovery of the electrical properties of some metals after a deformation at liquid-air temperature. It appeared that this recovery occurred in two steps, if the temperature of the recovery-treatment was increased. These results were quantitatively rather rough, since the time necessary to bring the wires to the recoverytemperature could not be neglected.

More accurate measurements have now been carried out on gold, silver and copper wires. The accompanying graph shows the results. For each metal the recovery-times were 15 and $45 \mathrm{~min}$. Vacant lattice sites can be accepted as the mechanism responsible for the increase of $\Delta p / p$. These vacant sites are produced during deformation and cause, together with dislocations, the change of the electrical properties. The change of the mechanical properties is assumed to be caused only by the pattern of dislocations.

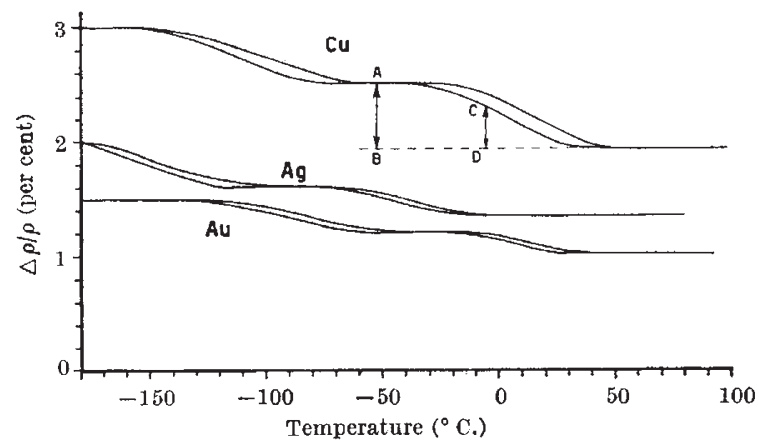

Recovery of $\Delta \rho / \rho$ may be produced by diffusion of the vacant lattice sites, during the recoverytreatment, to regions where their influence on the resistivity is negligibly small. These regions may be dislocations or the grain- or mosaic-boundaries. With this assumption it can be shown that the average concentration of vacant lattice sites in a grain, $\bar{c}$, is, to a first approximation (for times not too small), given as a function of time by

$$
\frac{\bar{c}}{c_{0}}=\frac{6}{\pi^{2}} \exp \left\{-\pi^{2} \frac{D t}{R^{2}}\right\},
$$

where $c_{0}$ is the concentration for $t=0, D$ is the diffusion coefficient, and $R$ is the radius of a sphere, which is assumed to be the shape of the grain or mosaic.

The relation between one time $(t)$ and one temperature $(T)$, which gives a certain value of $\bar{c} / c_{0}$, is

$$
t=A \exp [Q / k T],
$$

as follows from (1); $A$ is given by

$$
A=\frac{R^{2}}{\pi^{2} D_{0}} \ln \left\{\begin{array}{cc}
6 \\
\frac{\pi^{2}}{c}
\end{array}, \stackrel{c_{0}}{c}\right\} .
$$

$Q$ is the activation energy for one jump of the vacant lattice site; $D_{0}$ is a constant in $D=D_{0} \exp \{-Q / k T\}$.

These results can be applied to the experiments, since $c_{0} / c^{-}$corresponds with the relation $A B / C D$ of the recovery-curves. It is thus assumed that the extra-resistivity is proportional to the average concentration of vacancies.

The following results were obtained:

(1) The value of $Q$ can be calculated. This value appears to be a constant for one step of a certain recovery-curve. When the activation energy for the first step is called $Q_{1}$, and when $Q_{2}$ represents this energy for the second one, it was found that:

$\begin{array}{lcc} & Q_{1} \text { in } \mathrm{eV} . & Q_{2} \text { in } \mathrm{eV} . \\ \text { Gold } & 0 \cdot 29 & 0.69 \\ \text { Silver } & 0.18 & 0.65 \\ \text { Copper } & 0.20 & 0.88\end{array}$

This suggests that the mechanism of the second step is indeed the diffusion of single vacant lattice sites. It is possible that the first recovery step is: produced by the diffusion of special aggregates of vacant lattice sites.

(2) When the value of $A$ is plotted against. $\ln \left\{\frac{c_{0}}{\bar{c}} \cdot \frac{6}{\pi^{2}}\right\}$, a straight line is obtained for gold and copper. This relation is predicted by the diffusion formula (3).

(3) With the experimental value of $C=R^{2} / \pi^{2} D_{0}$ an estimate can be made of $R$. It was found that, for gold and copper, $R$ is of the order of $1 \mu$.

I wish to thank the Delfts Hogeschool Fonds for financial support.

\section{J. A. Manintveld}

Laboratorium voor Technische Physica, Delft.

Nov. 20.

${ }^{1}$ Molenaar, J., and Aarts, W. H., Nature, 166, 690 (1950).

${ }^{2}$ Druyvesteyn, M. J., and Manintveld, J. A., Nature, 168, 868 (1951).

\section{Striations on Prism Faces of Quartz}

STRIATIONS perpendicular to the $Z$-axis frequently occur on prism faces of quartz. They are often attributed to an 'oscillatory combination' of the prism and adjacent rhombohedron faces; but although this sometimes undoubtedly occurs (leading to tapered and 'candle' crystals), we believe that it is not the correct explanation of striations on welldeveloped crystals. A study of the surface topography of a number of natural crystals and a few synthetic crystals shows that the striations can be attributed to a difference in the rate of spreading of growth sheets parallel and perpendicular to the $Z$-axis.

The shape of the growth sheets on the prism faces of natural crystals is a parallelogram with two sides perpendicular to the $Z$-axis. The successive generation of growth sheets, from nuclei which frequently lie on the edge of the face or along a twin boundary intersecting the face, leads to the formation of lowangle growth pyramids. Fig. 1 is a photograph of these pyramids ( $Z$-axis vertical). Two sides of the pyramids are much steeper and longer than the other sides, owing to the differential rate of growth parallel and perpendicular to the $Z$-axis. The steeper sides thus give rise to striations, although their gradient is at most $15^{\circ}$, that is, considerably smaller than the angle of $38^{\circ}$ required by the oscillatory-combination theory. This explanation of the striations has also been given by A. F. Seager ${ }^{1}$. 